\section{Computer Control of Transmission Electron Microscopes: \\ Possibilities, Concepts and Present Limitations}

E. Völki, L.F. Allard, T.A. Dodson and T.A. Nolan

Oak Ridge National Laboratory

The electron microscope laboratory at the High Temperature Materials Laboratory in Oak Ridge National Laboratory runs essentially film free. This is possible due to the use of TV-rate and slow-scan CCD cameras together with fast desktop computers connected through Ethernet to network servers for image storage, and a variety of hard copy output devices. In our experience, the functionality of a film-free laboratory goes beyond the simple replacement of film material with some other storage media. For example, the time and effort to produce a final hardcopy image has been reduced effectively from several hours to several minutes. At the same time, data accuracy has increased due to the high linearity of the CCD cameras and data safety is improved due to automated nightly backups.

The present all-digital setup of our TEMs has already changed the routines on how a TEM is run. Two situations occur regularly: 1) one microscopist moves back and forth between the TEM and the computer and 2) two microscopists run the computer and/or TEM as a team. Unfortunately, the tasks of recording an image and running the microscope are still very much separated. Procedures such as stage movements, microscope alignment and many other routines are run from the microscope. Images are recorded and immediately processed at the computer to refine data. Our intention is to eliminate this separation and to optimize the usage of electron microscopes in general.

At our Hitachi HF2000 TEM, a Macintosh Quadra 950 with a 80 MFlop array processor is connected through an RS232 interface with the internal computer of the microscope as sketched in figure 1. A partial list of accessible functions is displayed in figure 3. This approached is, of course, not new.' Some routines are already controlled directly from the computer. For example, the software package DigitalMicrograph allows an automated fine-tuning of the microscope on a suitable specimen area using beam deflection controls. ${ }^{2}$ We have gone one step farther than partial microscope control and implemented all accessible microscope functions into DigitalMicrograph, i.e., the software that runs the CCD camera (and/or TV-camera) also runs the electron microscope.

The actual use of complex microscope control functions reveals major hardware problems which are present to some degree in almost every modern microscope. The primary obstacle is the response time of the microscopes to an external command. On the HF2000, the response time ranges between 0.2 and 0.3 seconds for a single command. For complex microscope control functions other than setting or receiving a single parameter - we find that, on the average about 10-20 SET- and GET-commands are necessary. To the human operator. those control functions should work without noticeable delay. Assuming a time delay of $<0.2$ seconds is not noticeable, we conclude that the average response time of the microscope to a single SET-GET command should be $<10 \mathrm{~ms}$.

The response-time of the beam shutter is another major obstacle. The shortest practical exposure time our present mechanical TEM beam shutter can handle is $>0.2 \mathrm{sec}$. We found that in using complex microscope control functions for analytical applications (such as EDS elemental profile mapping) it is essential to locate the position of the fully convergent electron beam. With our present beam shutter, the position can be located only for very small beam currents. For normal or high beam currents as used for analytical work, the CCD



Service contracts are expensive. Are you getting the service you paid for? Call the Materials Analytical Services' EM SERVICE GROUP before you sign another Service Contract.

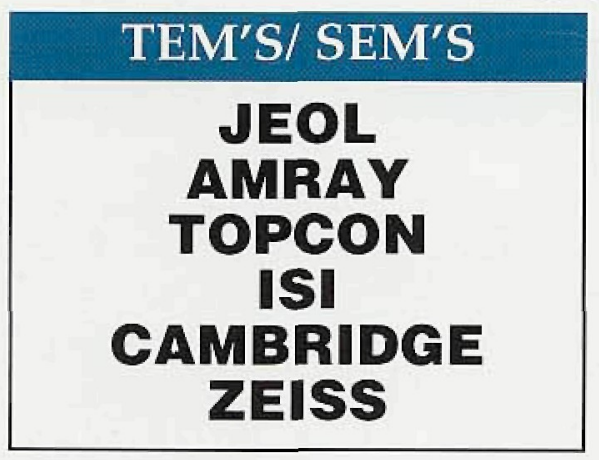

WE SERVICE

COMPUTER CONTROLLERS $X$-RAY SPECTROMETERS BEAM BLANKERS WATER CHILLERS

\begin{tabular}{|c|c|c|}
\hline \multicolumn{2}{|c|}{ ULTRAMICROTOMES } & PREP EQUIPMENT \\
\hline DUPONT & $\mathbf{R M}$ & VACUUM COATERS \\
\hline MT-1 & MT-5000 & PUTTER COATERS \\
\hline MT-2 & MT-6000 & MECHANICAL PUMPS \\
\hline MT-2B & MT-7000 & TURBO PUMPS \\
\hline$M T-2 C$ & CF & LASMA \\
\hline $\begin{array}{l}\text { Contra } \\
\text { Emmg } \\
\text { Reasor } \\
\text { Factor }\end{array}$ &  & \% $\mathrm{AN}$ \\
\hline $1-800$ & 2451 & \# \\
\hline
\end{tabular}

3597 Parkway Lane $\cdot$ Suite 250 - Norcross, Georgia 30092 • 770-448-3200 • FAX 770-368-8256 or 616 Hutton Street - Suite 101 - Raleigh, North Carolina 27606 • 919-829-7041 • FAX 919-829-5518 
chip becomes oversaturated, i.e., the position cannot be evaluated exactly and artifacts are created in successive images. We consider a beam shutter that allows exposure time of $<20 \mathrm{~ms}$ sufficient, and we are considering substituting a fast electromechanical device for the present mechanical shutter.

For the future we hope that manufacturers of electron microscopes will drastically improve the capability for external computer control of their microscopes. Practice has shown that present microscopes are far from being satisfactory for external computer control for the following reasons: 1) not all microscope functions are accessible (some functions are still analog only), 2) the response time of the microscopes is too slow (by about a factor of 1000), 3) the beam shutter in a TEM may be too slow, and 4) the SET-GET commands vary for different microscopes. Standard command sets would improve the potential to integrate every manufacturers instruments into the digitally controlled microscopy environment of the future. ${ }^{3}$

1. O. Krivanek et al., Proc. MSA (1993) 546

2. Gatan Inc., 6678 Owens Drive, Pleasanton, CA 94588

3. Research sponsored in part by the Laboratory Directed R\&D Program of Oak Ridge National Laboratory, managed for the DOE by Martin Marietta Energy Systems, Inc. under contract DE-AC05-840R21400 and supported by an appointment (E.V.) to the Oak Ridge National Laboratory Postdoctoral Research Program administered by the Oak Ridge Institute for Science and Education.

Reprinted, with permission, from the JMSA Proceedings of Microscopy and Microanalysis, 1996.


3

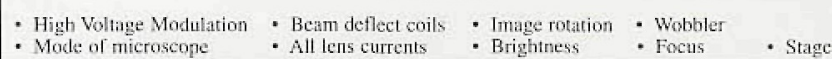

Figure 1. - External control of the Hitachi HF2000 TEM relies on the exchange of proprietary commands over a RS-232-C serial line. This imposes great transmission delays in addition to interpretation delays.

Figure 2. - External control of the TEM should rely on the exchange of standardized commands over a high speed interface such as HIPPI, ATM, or FDDI. In addition, the interpreter should be designed for high-speed command execution. Finally, actual analog status changes should be returned to the controlling computer

Figure 3 - Partial list of microscope functions implemented in DigitalMicrograph to run camera and HF2000 microscope. These microscope functions are building blocks for complex programs that add functionality to microscope and simplify TEM microscopy.

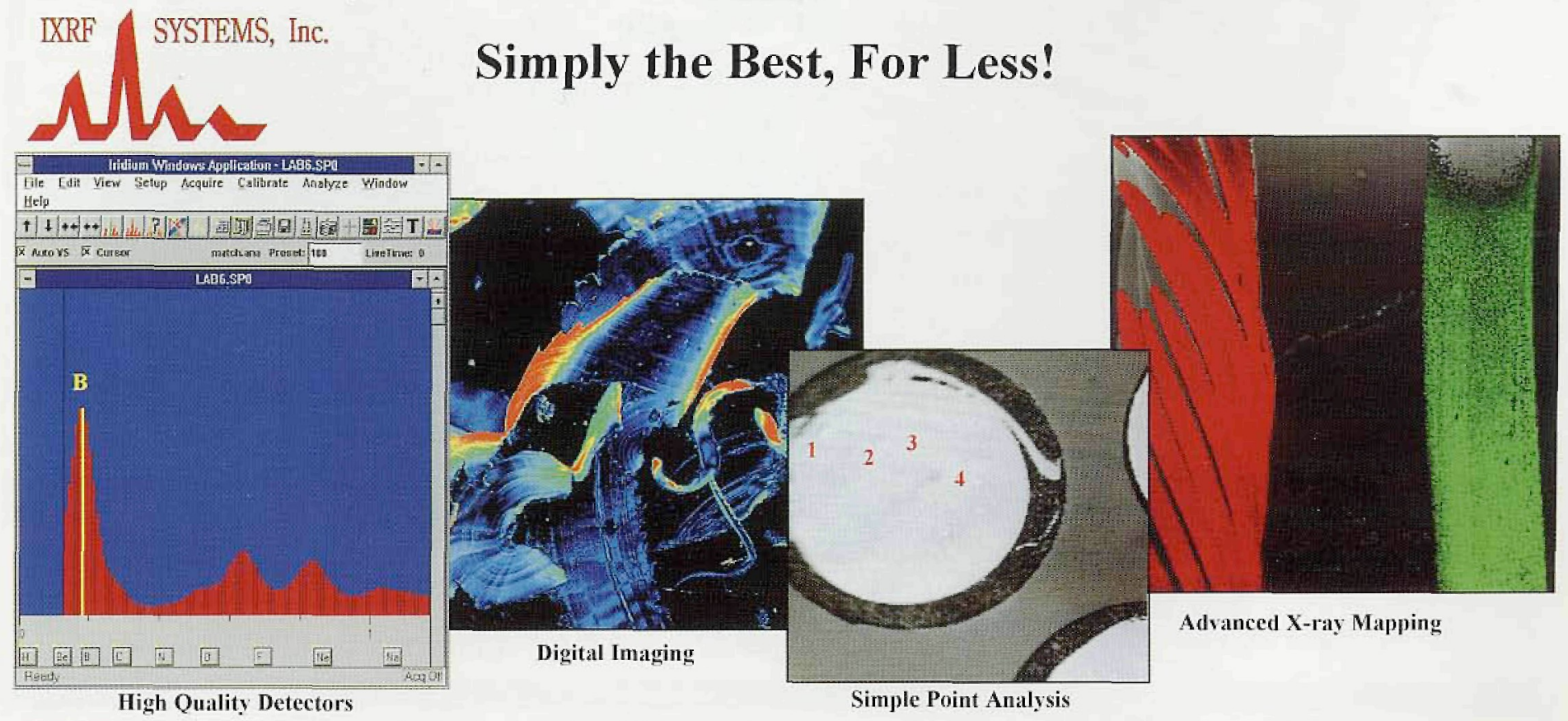

IXRF Systems. Inc. offers Microsoft Windows ${ }^{\text {TM }}$ EDS/Inaging systems for less than half the price of the leading EDS companies. We have the simplest yet most powerful EDS analysis package available. Our Phase Analysis. Auto Point Analysis, and Feature Analysis are just a few features which makes our Digital Imaging more advanced than the other larger EDS companies. Our Quantitative X-ray Mapping capabilities are second to none! Each system comes complete with Pentium PC's. installation/training, and color printer. IXRF Systems, Inc. has been labeled the "Fastest growing EDS company". Although IXRF Systems. Ine. is a small company. compared to the larger EDS manufacturers. our staff carries over 20 years of working and designing EDS/Imaging systems for the larger EDS companies. We will replace your existing system or provide new detectors for complete new systems. Call and request our information package to sce for yourself why we are SIMPLY THE BEST!

IXRF Systems, Inc. - 15715 Brookford Dr. - Houston, TX 77059 - Tel.: (713)286-6485 - Fax: (713)286-2660 